Publ. Astron. Soc. Aust., 1997, 14, 77-80

\title{
The Dark Blue Compact Dwarf Galaxy NGC 2915
}

\author{
Gerhardt R. Meurer \\ Department of Physics and Astronomy, The Johns Hopkins University, \\ Baltimore, MD 21218, USA \\ meurer@pha.jhu.edu \\ Received 1996 September 3, accepted 1996 October 10
}

\begin{abstract}
Recent results on NGC 2915, the first blue compact dwarf galaxy to have its mass distribution modelled, are summarised. NGC 2915 is shown to have $\mathrm{H}_{\mathrm{I}}$ well beyond its detected optical extent. Its rotation curve is well determined and fit with maximum disk mass models. The dark matter halo dominates the mass distribution at nearly all radii, and has a very dense core compared to those of normal galaxies. High-mass star formation energises the $\mathrm{H}_{\mathrm{I}}$ in the centre of the galaxy, but appears to be maintained in virial equilibrium with the dark matter halo. The implications of these results are briefly discussed.
\end{abstract}

Keywords: galaxies: individual (NGC 2915) — galaxies: kinematics and dynamics — dark matter

\section{Introduction}

Dark matter (DM) is a ubiquitous constituent of the universe; galaxies of all morphologies are now known to contain it. However, most studies of the distribution of DM about galaxies are weighted towards spiral, irregular and dwarf irregular (dI) galaxies. This is because these often have extended rotating $\mathrm{H}_{\mathrm{I}}$ disks which act as excellent dynamical tracers out to the large radii where DM dominates. There is one type of gas-rich galaxy that has been largely ignored in DM studies: the blue compact dwarf (BCD) class. In the optical, these galaxies are characterised by blue colours, high surface brightnesses, and sharp narrow emission lines: the hallmarks of intense massive star formation. In contrast, dI galaxies have similar luminosities, colours and gas contents, but much lower surface brightnesses and larger sizes. Although DM has been inferred from the dynamics of BCDs (Viallefond \& Thuan 1983), the study I describe presents the first detailed mass model decomposition of a BCD rotation curve. Whatever the reasons for neglecting BCDs in the past, I hope this study demonstrates that BCDs are well worthy of further attention, and that they may tell us something new about DM halo properties of galaxies.

The BCD galaxy NGC 2915 has a low surface brightness exponential radial surface brightness profile at large radii, and a high surface brightness blue core at its centre (Meurer, Mackie \& Carignan 1994). Although its appearance is somewhat peculiar (Sérsic, Bajaja \& Colomb 1977), its optically determined properties are not particularly exciting compared to those of other spectacular examples of the BCD class (e.g. IZw18, IIZw40, NGC 1569, NGC 1705). Becker et al. (1988), in a study of bright southern galaxies, showed that NGC 2915 is extended in $\mathrm{HI}$, has a double-horned velocity profile, and a large $M_{\mathrm{HI}}$ for its luminosity. It is these properties that made us decide to observe the galaxy with the Australia Telescope Compact Array (ATCA); extended $\mathrm{H}$ I distributions invariably reveal the DM halo of galaxies. Some important properties of NGC 2915 are summarised in Table 1. Details of our Hi study of NGC 2915 can be found in Meurer et al. (1996). Here I will present the main results and their implications.

Table 1. Properties of NGC 2915

\begin{tabular}{lcl}
\hline Quantity & Value & Comments \\
\hline$D$ & $5 \cdot 3 \pm 1 \cdot 6 \mathrm{Mpc}$ & Distance $^{\mathrm{a}}$ \\
$M_{B_{T}}$ & $-15 \cdot 90 \mathrm{mag}$ & Absolute magnitude \\
$R_{\mathrm{Ho}}$ & $2 \cdot 93 \mathrm{kpc}$ & Holmberg radius \\
$R_{\mathrm{HI}}$ & $14 \cdot 9 \mathrm{kpc}$ & H I radius \\
$R_{\mathrm{HI}} / R_{\mathrm{Ho}}$ & $2 \cdot 7$ & \\
$M_{\mathrm{HI}}$ & $9 \cdot 58 \times 10^{8} M_{\odot}$ & H I mass \\
$M_{\mathrm{HI}} / L_{\mathrm{B}}$ & $2 \cdot 7\left(M / L_{\mathrm{B}}\right)$ & \\
\hline a Consistent with $H_{0}=50 \mathrm{~km} \mathrm{~s}^{-1} \mathrm{Mpc}^{-1}$ \\
b Radius of isophote having B surface brightness of $26 \cdot 5 \mathrm{mag}$ \\
arcsec \\
${ }^{-2}$ Radius having face-on column density $N_{\mathrm{HI}}=5 \times 10^{19} \mathrm{~cm}^{-2}$
\end{tabular}

\section{H I Properties}

In Figure 1 the optical (I band) and $\mathrm{H}$ I morphologies of NGC 2915 are compared. The $\mathrm{H}_{\mathrm{I}}$ is very extended relative to the optical and has a barred spiral morphology. The optical counterpart corresponds well in size and position angle to the bar (and does not extend beyond it, to our detection limits). The velocity field is dominated by the rotating disk, but also shows the oval distortion due to the bar: the kinematic major and minor axes are not aligned. The disk is quiescent beyond 

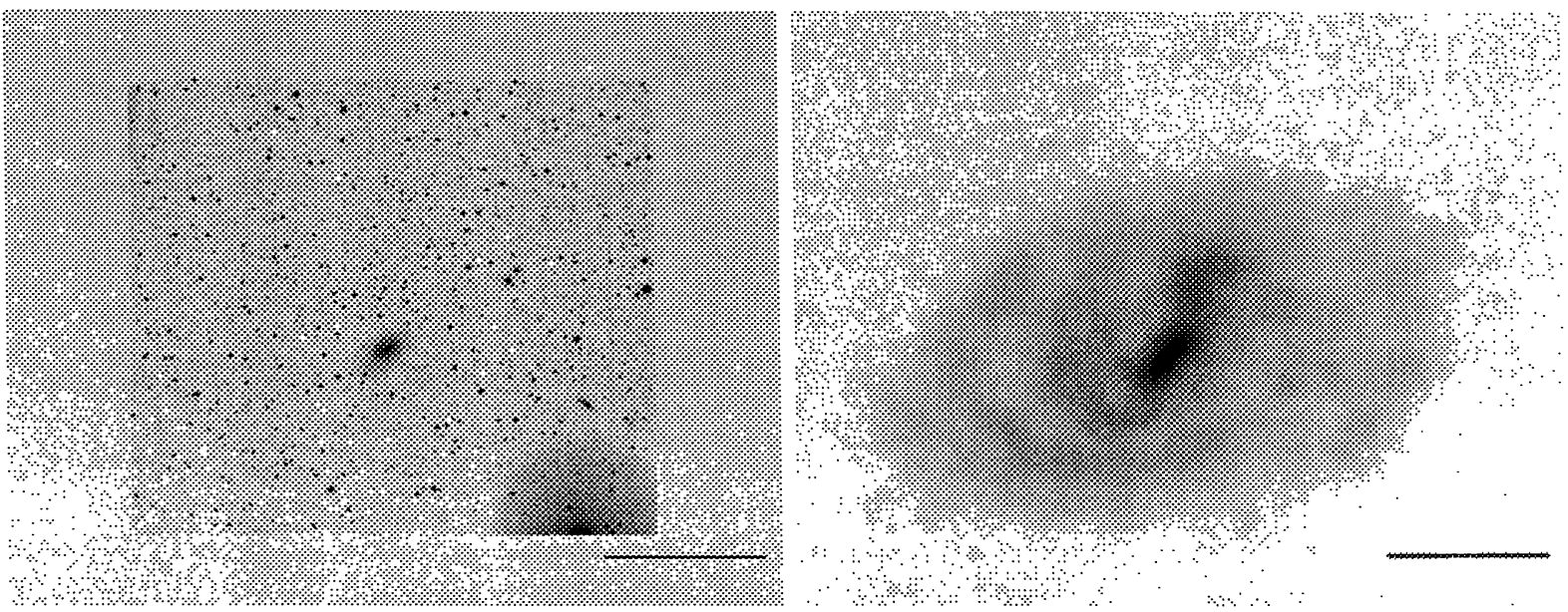

Figure 1-NGC 2915 in the I band (left) from an $800 \mathrm{~s}$ integration using the Anglo-Australian Telescope, and in total $\mathrm{HI}$ emission (right) from our ATCA observations. North is up, east is to the left, and the scalebars are $5^{\prime}=7 \cdot 7 \mathrm{kpc}$ long.

the optical extent, having a velocity dispersion of $\sigma \approx 8 \mathrm{~km} \mathrm{~s}^{-1}$, which is common for spiral galaxies. Near the centre of the galaxy the velocity dispersion reaches $\sigma \approx 40 \mathrm{~km} \mathrm{~s}^{-1}$, and the $\mathrm{H}$ I line is often split. The broad and split line profiles are associated with massive star-forming regions and expanding bubbles that are prominent in the $\mathrm{H} \alpha$ image of Marlowe et al. (1995). Thus star formation is energising the neutral interstellar medium in the centre of NGC 2915.

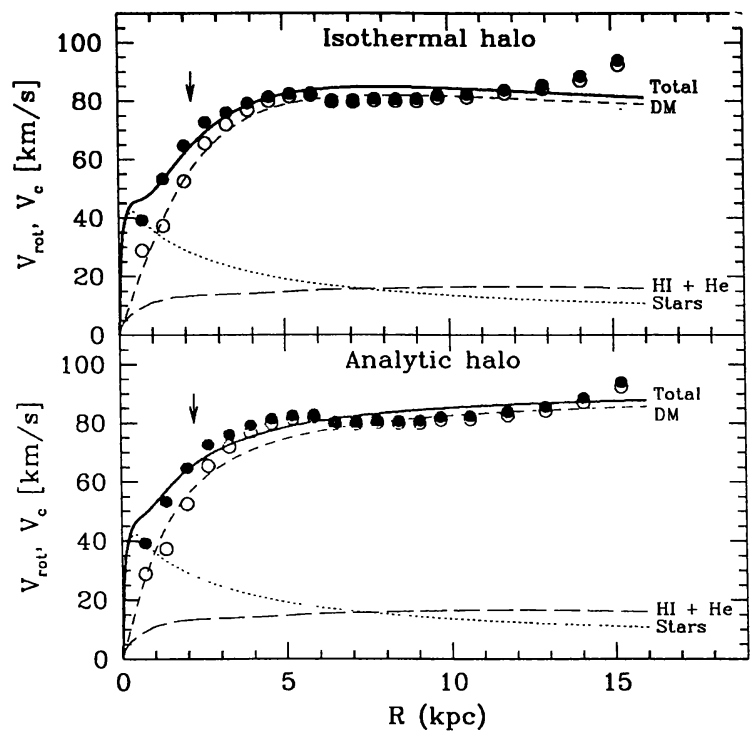

Figure 2-Rotation curve of NGC 2915 compared to two mass models. Raw rotation velocities $V_{\text {rot }}$ are shown with open symbols, while pressure support corrected circular velocities $V_{c}$ are shown as closed symbols. The Holmberg radius $R_{\mathrm{Ho}}$ is indicated with the vertical arrows.

The rotation velocity $V_{\text {rot }}$ curve of NGC 2915 is shown in Figure 2. The raw $V_{\text {rot }}$ measurements were corrected for pressure support (asymmetric drift) to obtain circular velocities $V_{\mathrm{c}}$, which are also shown.
The optical portion of NGC 2915 is wholly contained in the rising portion of the rotation curve. This is where rotational shear is minimised, hence where molecular clouds have the longest lifetime and star formation should be most efficient. The rotation curve levels off shortly beyond the Holmberg radius $R_{\text {Ho }}$, remains approximately flat out to about $10 \mathrm{kpc}$, and then rises again out to the last measured point $(R=15 \cdot 2 \mathrm{kpc})$.

\section{Dark Matter Halo Properties}

Figure 2 shows maximum disk mass model fits to NGC 2915's $V_{\mathrm{c}}$ curve. There are three components to the mass models: (1) a stellar disk parametrised by its mass-to-light ratio, here set at $M_{*} / L_{\mathrm{B}}=$ $1 \cdot 2\left(M / L_{\mathrm{B}}\right)_{\odot}$ (the maximum allowed by the innermost $V_{\mathrm{c}}$ measurement); (2) a neutral gaseous disk whose mass distribution is determined directly from the ATCA data; and (3) a spherical dark matter halo parametrised by a velocity dispersion $\sigma_{0}$, which determines the level of the flat part of the rotation curve, and the core radius $R_{c}$, which determines the location of the knee in the rotation curve. These parameters yield the central DM density $\rho_{0}$. Two types of DM halo were fitted: (a) a non-singular isothermal sphere, and (b) an analytic DM density distribution:

$$
\rho(R)=\frac{\rho_{0}}{1+\left(R / R_{\mathrm{c}}\right)^{2}} .
$$

Both forms produce flat $V_{\mathrm{c}}$ curves at large $R$, but have different density distributions and different scaling relations between $R_{\mathrm{c}}, \rho_{0}, \sigma_{0}$ and the asymptotic $V_{\mathrm{c}}$ at $R=\infty$ (Lake, Schommer \& van Gorkom 1990; Binney \& Tremaine 1987). The parameters of the two fits are given in Table 2. The isothermal halo does not fit our data as well as the analytic halo. However, many fits in the literature 
use this form to model the DM halo, so we include its fit for comparison.

Table 2. DM halo fits

\begin{tabular}{lcc}
\hline & \multicolumn{2}{c}{ Halo model } \\
Parameter & Isothermal & Analytic \\
\hline$R_{\mathrm{c}}[\mathrm{kpc}]$ & $2 \cdot 8 \pm 0 \cdot 2$ & $1 \cdot 2 \pm 0 \cdot 2$ \\
$\sigma_{0}\left[\mathrm{~km} \mathrm{~s}^{-1}\right]$ & $51 \cdot 7 \pm 0 \cdot 9$ & $41 \cdot 0 \pm 1 \cdot 1$ \\
$\rho_{0}\left[M_{\odot} \mathrm{pc}^{-3}\right]$ & $0 \cdot 058 \pm 0 \cdot 009$ & $0 \cdot 10 \pm 0 \cdot 02$ \\
$M_{\mathrm{T}} / L_{\mathrm{B}}\left(R_{\mathrm{Ho}}\right)$ [solar units] & $10 \cdot 0$ & $9 \cdot 6$ \\
$M_{\mathrm{T}} / L_{\mathrm{B}}\left(R_{\mathrm{HI}}\right)$ [solar units] & 66 & 76 \\
\hline
\end{tabular}

The important things to note about the mass model fits are: (1) The neutral gaseous disk is more massive than the maximum stellar disk, hence NGC 2915 is not a very evolved galaxy. (2) DM dominates at nearly all radii. For the analytic model, $M_{T} / L_{B}=76$ within the last measured point, making NGC 2915 along with DDO 154 (Carignan \& Beaulieu 1989) the darkest known disk galaxies. (3) The DM central velocity dispersion $\sigma_{0}$ is equal to or greater than the $\sigma_{\mathrm{HI}}$ in the centre. Thus although the $\mathrm{H}_{\mathrm{I}}$ is violently energised by star formation, it is still in virial equilibrium with the DM halo. This suggests that the DM halo may be involved in regulating NGC 2915's star formation: if the star formation rate gets too high it will over-energise the ISM so that $\sigma_{\mathrm{HI}} \gg \sigma_{0}$, then the ISM will be blown out of the core and star formation will be halted. (4) The halo core is an order of magnitude denser than those found in 'normal' disk galaxies. This is illustrated in Figure 3, which plots $\rho_{0}$ versus $M_{B}$ for disk galaxies of various types.

\section{NGC 2915's Oddness and How to Put It in Its Place}

NGC 2915 is an interesting galaxy because of its extremely odd qualities: its large DM content, the contrast between its optical and $\mathrm{H}$ I properties, and the high $\rho_{0}$ of its DM halo. It is also recalcitrant compared to normal galaxies because: (1) it does not obey the Tully-Fisher relationship; (2) its neutral gas disk is $\sim 10 \times$ under-dense for it to have the star formation observed in the core (Kennicutt 1989); (3) similarly, the outer disk is $Z 2 \times$ under-dense for it to have the spiral arms that are observed. This suggests that some of its DM is in the disk, perhaps in a molecular form. These properties are discussed more fully in Meurer et al. (1996).

NGC 2915 is an important galaxy because it is not that odd compared to other BCDs which often have very extended $\mathrm{HI}$ and similar radial profiles of H I surface density (often centrally peaked) and velocity dispersion, as well as flat rotation curves at large radii (Taylor et al. 1994; 1995; Hunter, O'Connell \& Gallagher 1994; Meurer 1994; Brinks \& Klein 1988; Viallefond \& Thuan 1983). This suggests that BCDs as a class have fundamental structural differences from dI and spiral galaxies,

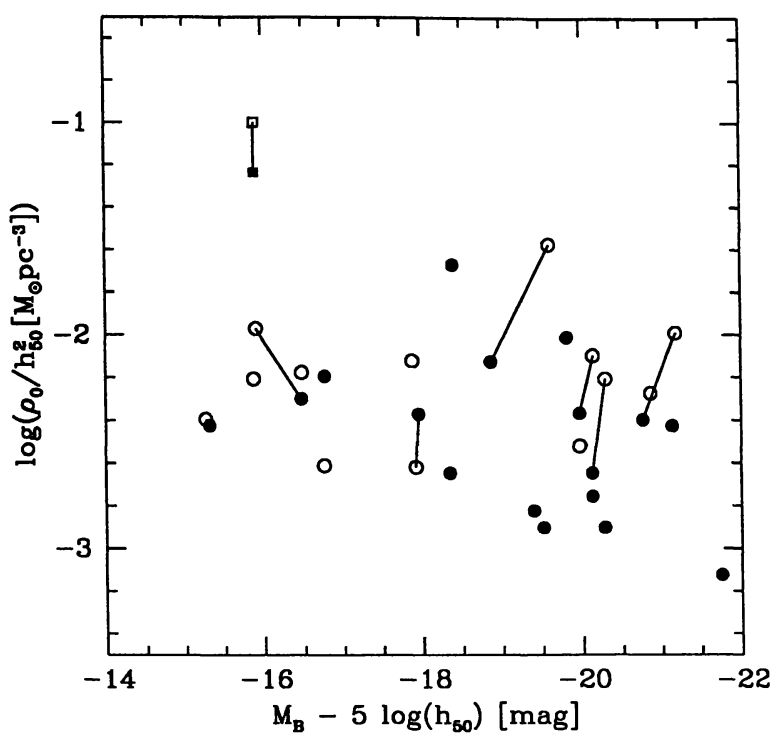

Figure 3-DM halo central density $\rho_{0}$ compared to absolute magnitude, where both quantities have been corrected (to first order) for the different distance scales. Here $h_{50}=H_{0} /\left(50 \mathrm{~km} \mathrm{~s}^{-1} \mathrm{Mpc}^{-1}\right)$ is the dimensionless Hubble constant used in the individual studies. Circles represent fits to $\mathrm{dI}$ and spiral galaxies, while the squares represent the fits to NGC 2915 presented here. Solid symbols show isothermal sphere halo model fits, hollow symbols show analytic halo model fits. Connected points represent different halo models fit to the same obect. The dI and spiral data were taken from Puche \& Carignan (1991), Begeman, Broeils \& Sanders (1991) and Sanders (1996 and references therein).

and NGC 2915 is only odd because it is the first BCD examined in such detail.

It is therefore important to do further detailed $\mathrm{H}_{\mathrm{I}}$ studies of BCDs. There are two issues in particular that need to be addressed. First, if the abnormally high $\rho_{0}$ of NGC 2915 is common to BCDs, then transformations between $\mathrm{BCD}$ and $\mathrm{dI}$ morphologies could not be accomplished by turning starbursts on and off. Fundamental structural changes to the DM distributions would also have to occur, and this seems hardly likely. Such a result would make it hard or impossible to reconcile BCDs and dIs to some common evolutionary scenario. Second, the near equality between $\sigma_{\mathrm{HI}}$ and $\sigma_{0}$ of the DM in NGC 2915 suggests that the star formation is held in equilibrium with the DM halo. This is contrary to the common perception of explosive starbursts dominating the star formation and dynamics of BCDs. Further large-sample comparisons of starburst dynamics and halo properties are needed to determine if there is a feedback mechanism operating between the two structures.

There may be a more mundane explanation for NGC 2915's peculiarities. Meurer et al. (1994) determined $D$ from the colour magnitude diagram of NGC 2915's bright stars and star clusters. If it is more distant by a factor of $\sim 2$ (i.e. larger than their 
stated $D$ uncertainty) many of its odd properties would disappear. A better $D$ determination and a search for faint stellar light associated with the $\mathrm{H}$ I disk (both could be done with the Hubble Space Telescope) may reduce NGC 2915's odd qualities to its peculiar motion relative to the Hubble flow.

So what is the relevance of NGC 2915 to the multibeam survey? NGC 2915 was painstakingly mapped by Becker et al. (1988) with the Parkes radio telescope, pointing at 19 positions plus sky, and requiring over three hours of telescope time. It is these observations that first indicated NGC 2915's huge $\mathrm{H}_{\mathrm{I}}$ extent. The multibeam survey is bound to uncover similar galaxies with extended $\mathrm{H}_{\mathrm{I}}$ disks using integration times of only a few minutes. This study illustrates what follow-up observations with the ATCA or Very Large Array may reveal about the DM distribution and dynamics of such galaxies.

\section{Acknowledgments}

I thank the coauthors of my NGC 2915 studies, Sylvie Beaulieu, Claude Carignan, Ken Freeman, and Glen Mackie, for all the work they did. Sylvie Beaulieu and Chris Mihos kindly read and commented on the paper before submission. The American Astronomical Society generously supplied me with a travel grant to attend the multibeam workshop. I am grateful to the organisers of the workshop for arranging local funding, and to Lister
Staveley-Smith and Jessica Chapman for opening their home to me during my stay in Sydney.

Becker, R., Mebold, U., Reif, K., \& van Woerden, H. 1988, A\&A, 203, 21

Begeman, K. G., Broeils, A. H., \& Sanders, R. H. 1991, MNRAS, 249, 523

Binney, J., \& Tremaine, S. 1987, Galactic Dynamics (Princeton Univ. Press)

Brinks, E., \& Klein, U. 1988, MNRAS, 231, 63P

Carignan, C., \& Beaulieu, S. 1989, ApJ, 347, 760

Hunter, D. A., O'Connell, R. W., \& Gallagher, J. S. 1994, AJ, 108,84

Kennicutt, R. C. 1989, ApJ, 344, 685

Lake, G., Schommer, R. A., \& van Gorkom, J. H. 1990, ApJ, 320, 493

Marlowe, A. T., Heckman, T. M., Wyse, R. F. G., \& Schommer, R. 1995, ApJ, 438, 563

Meurer, G. R. 1994, in ESO/OHP Workshop on Dwarf Galaxies (ESO Conference \& Workshop Proceedings no. 49), ed. G. Meylan \& P. Prugniel (Garching: ESO), p. 351

Meurer, G. R., Mackie, G., \& Carignan, C. 1994, AJ, 107, 2021

Meurer, G. R., Carignan, C., Beaulieu, S., \& Freeman, K. C. 1996, AJ, 111, 1551

Puche, D., \& Carignan, C. 1991, ApJ, 378, 487

Sanders, R. H. 1996, ApJ, 473, 117

Sérsic, J. L., Bajaja, E., \& Colomb, R. 1977, A\&A, 59, 19

Taylor, C. L., Brinks, E., Pogge, R. W., \& Skillman, E. D. 1994, AJ, 107, 971

Taylor, C. L., Brinks, E., Grashuis, E., \& Skillman, E. D. 1995, ApJS, 99, 427

Viallefond, F., \& Thuan, T. X. 1983, ApJ, 269, 444 